\title{
Exploring the influence of a cooling treatment on quality of life in patients with chronic venous disease
}

\section{Teresa J Kelechi \\ Martina Mueller \\ Mohan Madisetti \\ Margie A Prentice \\ Mary J Dooley}

College of Nursing, Medical

University of South Carolina,

Charleston, SC, USA

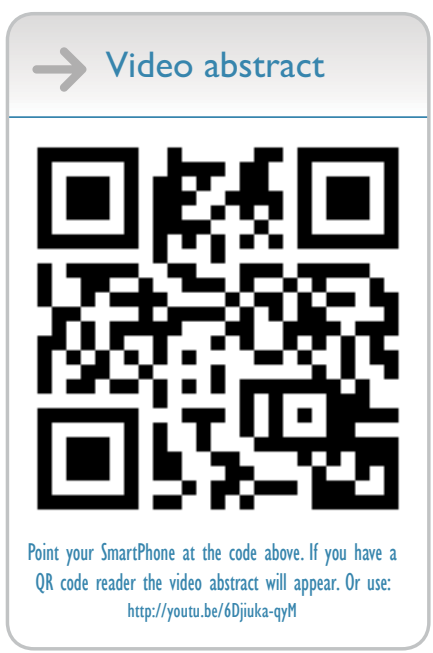

Correspondence: Teresa J Kelechi College of Nursing, Medical University of South Carolina, 99 Jonathan Lucas Street, MSC 160, Charleston SC 29425, USA

$\mathrm{Tel}+$ I 8437924602

Fax +I 8437922099

Email kelechtj@musc.edu
This article was published in the following Dove Press journal:

Chronic Wound Care Management and Research

22 May 2017

Number of times this article has been viewed

Purpose: The study aimed to evaluate the influence of a self-administered cooling intervention on quality of life (QOL) associated with chronic venous disease (CVD), stages clinical, etiological, anatomical, pathophysiological (CEAP) C4 (skin damage) and C5 (healed ulcer).

Study design and subjects: A sample of 276 individuals was randomized to receive a cooling $(n=138)$ or placebo control cuff $(n=138)$ applied to the leg skin affected by CVD. Both groups also received standard of care that included compression, leg elevation, and physical activity. QOL was measured with the venous insufficiency epidemiological and economic study (VEINES)QOL and symptom (Sym) subscale Questionnaire at 5 time points during the 9-month study. Relationships between treatment outcomes and demographics were analyzed.

Results: Cooling and control groups had significant increases from baseline in mean change VEINES-QOL scores (13.5 vs 12.8, $p<0.0001)$ and Sym scores (10.4 vs $6.7, p<0.0001)$. No significant difference was observed for VEINES-QOL between the groups $(\Delta=0.67, p=0.58)$; however, the difference was significant for Sym for cooling $(\Delta=3.7, p=0.015)$. Overall QOL improvements were significant for females compared to males $(p<0.001)$, not employed fulltime $(p<0.001)$, living in rural areas $(p<0.002)$, and less effective for larger calf circumference ( $p=0.042$ ). For age groups $\geq 65$ years, cooling produced significant improvements in QOL (10.8 vs $4.5, p<0.0004)$; the control group symptoms worsened during the study $(-1.0$ vs $8.1, p<0.0001)$. Conclusion: This cooling intervention improved QOL. The greatest improvements were observed in older individuals, females, those who were married, not working full time, and living in rural areas. CVD remains a poorly controlled chronic condition and has a major negative influence on QOL. Keywords: chronic venous disease, quality of life, cooling therapy, negative symptoms, cryotherapy, venous disorders

\section{Plain language summary}

The purpose of this study was to determine whether quality of life (QOL) was affected by the use of a cooling cuff to reduce negative symptoms of chronic venous disease (CVD) of the lower legs where the skin was previously ulcerated and/or damaged. Individuals participating in the study received one of two treatments: a cooling cuff made of gel or a "placebo" cuff filled with cotton. The cuffs were kept in the freezer until they were ready to be applied to the affected skin, starting daily for 30 minutes for 30 days, then twice-weekly for 3 months and then weekly for 3 months. Participants also wore compression hosiery during the day and elevated their legs each evening during the treatment. The VEINES Sym/QOL Questionnaire was used to measure QOL. We found that QOL improved in individuals using the cooling cuff compared to those using the cotton cuff; symptoms become worse in the latter group. Greater improvements were reported by females and those 65 years of age and older. Females and those older in age may be more positively susceptible to the effects of cooling to reduce negative symptoms of disease. 


\section{Introduction}

It is well established that chronic illness negatively affects quality of life (QOL); thus, it is not surprising that individuals with chronic venous disease (CVD) experience such negative consequences. ${ }^{1-3} \mathrm{CVD}$ is caused by venous hypertension that develops from perforator vessel and valvular incompetence, which lead to other anomalies of the venous vasculature, including varicosities, swelling, skin damage, and leg ulcers. ${ }^{4}$ Individuals with CVD report a myriad of symptoms such as pain, throbbing, itchiness, achiness, and heaviness. ${ }^{5,6}$ As the disease progresses, there are negative QOL effects on daily living, physical functioning and mobility, and psychological and social wellbeing reflected as depression, feelings of worthlessness, low mood, and social isolation. ${ }^{711}$ However, interventions designed specifically to improve QOL of individuals with CVD remain elusive.

There have been some improvements in QOL reported by patients with CVD receiving medications such as venoactive drugs including micronized purified flavonoid fraction, ${ }^{12}$ undergoing surgical treatment, ${ }^{13}$ or receiving ultrasound-guided foam sclerotherapy. ${ }^{14}$ Lower leg exercise studies demonstrate improvements in leg function, but there is limited efficacy on QOL outcomes. ${ }^{15}$ Even standard of care (ie, compression hosiery, leg elevation) coupled with venoactive drugs and endovenous thermal ablation has not consistently demonstrated QOL improvements. ${ }^{16}$ These findings underscore the need for behavioral interventions and therapies that reduce negative symptoms and improve QOL.

An exploratory objective of this randomized trial was to evaluate the influence of a self-administered cooling therapy on QOL in patients with the more severe stages of CVD, clinical, etiological, anatomical, pathophysiological (CEAP) classification system classes $\mathrm{C} 4$ (skin damage) and C5 (history of leg ulcers). Cooling has been shown to improve numerous acute and chronic conditions, ${ }^{17-19}$ but the influence on QOL in patients with a chronic condition such as CVD has not been established. Differences in age and sex were of particular interest, given that the disease disproportionately affects older females. Race and socioeconomic differences were also measured, as these QOL disparities are well established. QOL was measured with the CVD-specific VEINES-QOL instrument and subscale that provides an overall score of physical and psychosocial function. These exploratory aims sought to describe: 1) Differences in QOL total (VEINES-QOL) and subscale (VEINES-Sym) scores between the cooling and control groups, unadjusted and adjusted for covariates including sex, marital status, and residence (rural or urban). 2) Differences in race, employment status, body mass index (BMI), and leg circumference for participants $<65$ and $\geq 65$ years of age between the cooling and control groups. 3) Differences in QOL total (VEINESQOL) and subscale (VEINES-Sym) scores for participants $<65$ and $\geq 65$ years of age among and between the cooling and control groups.

\section{Study design and subjects}

In this 9-month randomized clinical trial cooling intervention, signals of efficacy on QOL were explored and personal demographic and clinical factors related to QOL findings were investigated. The study was approved by the Institutional Review Board of the Medical University of South Carolina, Charleston, SC, USA (Protocol No. 00008711), registered with ClinialTrials.gov (NCT0509599) and conducted at three sites in the Eastern US between September 2010 and December 2015. The population included patients diagnosed with CVD by the health care clinician or specialist. Subjects were randomized to receive a cooling cuff made of a flexible solid hydrogel or a placebo cuff filled with cotton batting, and the outcome of QOL was measured with the VEINES-QOL/Sym. The inclusion criteria were: 1) age 21 years and above; 2) english speaking; 3) ankle brachial index, a measure of arterial insufficiency, between 0.8 and $1.3 \mathrm{mmHg}$; 4) clinical manifestations CEAP C4 and $\mathrm{C} 5$; 5) understand protocol by passing 10 -question verbal test.

The seven CEAP clinical classes ${ }^{20}$ are shown in Table 1. The exclusion criteria were: 1) no diagnosis of venous insufficiency; 2) active leg or foot ulcer; 3) lower leg skin infection within past month; 4) surgical procedures performed on CVD affected leg in past 12 months; 5) comorbid conditions such

Table I CEAP clinical classification stages for CVD

\begin{tabular}{ll}
\hline Clinical stage classification & Description \\
\hline $\mathrm{C} 0$ & No visible or palpable disease \\
$\mathrm{Cl}$ & Telangiectasia or reticular veins \\
$\mathrm{C} 2$ & Varicose veins \\
$\mathrm{C} 3$ & Edema \\
$\mathrm{C} 4$ & Changes in skin and subcutaneous \\
& tissue: hemosiderin staining, eczema, \\
& lipodermatosclerosis, atrophe blance \\
$\mathrm{C} 5$ & Skin changes with healed ulcer \\
$\mathrm{C} 6$ & Changes in skin with active ulcer \\
\hline
\end{tabular}

Abbreviations: CEAP, clinical, etiological, anatomical, pathophysiological; CVD, chronic venous disease. 
as scleroderma, Raynaud's disease, lupus erythematosus, or lymphedema; 6) receiving chemotherapy; 7) loss of neurosensory or thermal skin sensation on the feet; 8) unable to wear compression wraps during waking hours; 9) not accessible by phone, email, or mail.

Patients were approached in the clinic by providers and recruited through public advertisements and word of mouth. Written informed consent was obtained from all participants after explaining the study purpose and procedures, frequency of visits, and compensation. A total of 347 patients with CVD were approached, 276 consented and were randomized to receive the cooling intervention $(n=138)$ or placebo control $(\mathrm{n}=138)$. Randomization was carried out using a stratified permuted block design with a computer-generated allocation scheme to assign eligible participants, stratified by site, ie, practice, wound center, and self-referral. Treatment allocation was unknown to both participants and study staff until after baseline measures were completed. The cuffs were designed to look identical in color and size in an attempt to blind the participant as much as possible.

\section{Group interventions}

Both groups received standardized instruction on the procedures. A 6-minute DVD of all study procedures including standard of care that included compression with $30 \mathrm{mmHg}$ leggings (JuxtaLite; CircAid by medi, San Diego, CA, USA), leg elevation with a 20 -inch foam wedge pillow, and how to perform proper skin hygiene and lower leg exercises was shown by the research assistant to each participant, followed by a question/answer period. A 10-item oral quiz was given and return demonstration by the participant on the use of study equipment (eg, use of skin thermometer to self monitor temperature, application of the cuff, recording of data on study logs) to determine mastery of content was conducted after baseline data were collected. The treatment and control groups received a cooling and placebo cuff, respectively, specially designed by Southwest Technologies (Kansas City, MO, USA) and were kept in the freezer until the time of use. The cooling cuff is made from a $3 / 4$ inch sheet hydrogel containing glycerin that prevents the material from freezing to a solid state. The sheet is encased in two polyethylene sleeves that prevent the cold hydrogel from directly contacting the skin so as to reduce the risk of frostbite. The thickness of the material was tested for its safety prior to use in the study, as available sheet gels were found to be too thin to effectively cool the skin. The material is malleable, allowing for conformity to various leg sizes and shapes. The cuff works by "pulling" heat from the superficial skin layers to reduce inflammation by slowing the abnormal metabolic activity associated with venous disease. Persistent, chronic inflammation damages the skin, predisposing it to ulceration. Cooling the skin thus prevents further skin damage and breakdown. ${ }^{18}$

The protocol consisted of application of the cuff during 30 minutes of leg elevation per the following regimen: daily in month 1 , twice weekly in months $2-3$, once weekly in months 4-6, and then as needed in months 7-9 in response to a $2^{\circ} \mathrm{C}$ skin temperature elevation above the recorded baseline skin temperature. Participants measured skin temperature before, after, and 12 hours after the intervention with a long-handled contact infrared thermometer (TempTouch $\AA$; Diabetica Solutions, San Antonio, TX, USA). These self monitoring data were recorded on a study log for the purpose of determining skin temperature changes over time.

\section{Measures and data collection}

After consenting and enrollment, patients were interviewed at the baseline visit (V1). Data included demographic characteristics such as age and sex, clinical data (health and medication history), and physiological data such as lower leg skin temperature and blood flow, and QOL.

To determine baseline, participants recorded on a standardized form the skin temperature of the affected leg each morning, measured $5 \mathrm{~cm}$ above the medial malleolus, for 28 days. The average of these measurements was considered the baseline skin temperature.

QOL was measured with the VEINES QOL/Sym Questionnaire $^{21}$ during an interview conducted at baseline V1, after month 1 (V2), month 3 (V3), month 6 (V4), and month 9 (V5). All data were entered into a web-based Research Electronic Data Capture (REDCap) management system. Over the course of the study, adherence to the cuff application was tracked via self-report on written logs. The logs were brought to each visit.

\section{VEINES QOL/Sym Questionnaire}

The VEINES QOL/Sym Questionnaire is a 26-item patientbased, disease-specific questionnaire designed for self-completion. Calculation of the total score included summing 25 of the 26 items that estimate daily living and psychological impacts of CVD (25 items) on QOL and symptoms (10 items) (VEINES-Sym subscale) including heavy legs, aching legs, swelling, night cramps, burning sensation, itching, throbbing, pain in the legs, restless legs, and numbness resulting from CVD. One of the items, Question 2 was used for descriptive purposes only and is not included in the overall score as it 
takes into account the time of day when symptoms are more intense. Psychometric evaluation of total VEINES-QOL and VEINES-Sym scores has demonstrated good validity and reliability in numerous studies of CVD, including in individuals with leg ulcers and deep vein thrombosis.

\section{Statistical analyses}

Sample size was determined for the exploratory outcome, total VEINES-QOL score. With 96 participants randomized to each of the two treatment groups for a total $\mathrm{N}$ of 192, we had $85 \%$ power to detect a 0.49 standardized effect size (eg, Cohen's $d$ ) between the groups. We assumed 20\% attrition based on data from our previous study conducted over 30 days ${ }^{22}$ however, for the final sample size, the inflation was increased to $30 \%$ due to additional dropouts over this longer study period ( 9 months). The sample size was subsequently inflated to account for drop-outs over the study period to a final $\mathrm{N}$ of 276 participants ( 138 subjects per group). Given the exploratory nature of the study, the significance level $(\alpha)$ was maintained at 0.05 and was not adjusted for multiple outcome variables being assessed or multiple comparisons within outcomes to protect against inflation of the false-positive error (Type I) that could result in an overly conservative conclusion.

For the comparisons between groups for continuous and categorical variables, pooled $t$-tests and $\chi^{2}$ tests were used, respectively. For all analyses that involved the examination of the effect of race on QOL, two participants were excluded in the "other race" category; comparison was restricted to White and Black. While detailed distributions of demographic and clinical measures are reported in Table 2, for some analyses, response categories were collapsed, for example, marital status and employment status were dichotomized. Marital status was defined as married vs not married which included never married, widowed, separated, or divorced. Employment was defined as employed (full or part-time) vs not employed (unemployed, retired, volunteer, student, or home-maker). Statistical analyses were conducted using SAS 9.4 (Cary, NC, USA).

All VEINES-QOL and VEINES-Sym total scores were obtained using Bland et al's ${ }^{23}$ intrinsic score method to allow for the comparison of scores over time.

All longitudinal models were analyzed using a mixed effects models approach with clustering by participant to control correlation among repeated measurements and allow for analysis of all subjects including those missing data at various visits. Dependent variables were the change from baseline for VEINES-QOL and VEINES-Sym at each visit over the study period of 9 months. Group (cooling vs control)
Table 2 Demographic features of sample by age and treatment group

\begin{tabular}{|c|c|c|c|c|c|}
\hline \multirow[t]{3}{*}{ Demographics } & \multicolumn{2}{|l|}{ Cooling } & \multicolumn{2}{|l|}{ Control } & \multirow[t]{3}{*}{$p$-value } \\
\hline & Age $<65$ & Age $\geq 65$ & Age $<65$ & Age $\geq 65$ & \\
\hline & $n=85$ & $n=53$ & $n=8 I$ & $n=57$ & \\
\hline Age (years) & $55.4 \pm 7.3$ & $74.3 \pm 6.3$ & $52.9 \pm 7.8$ & $72.2 \pm 6.2$ & - \\
\hline Sex & & & & & 0.690 \\
\hline Male & 38 (44.7\%) & 28 (52.8\%) & 36 (44.4\%) & 24 (42.1\%) & \\
\hline Female & 47 (55.3\%) & 25 (47.2\%) & 45 (55.6\%) & 33 (57.9\%) & \\
\hline Race & & & & & $0.00 \mathrm{I}^{\mathrm{a}}$ \\
\hline White & 35 (4I.2\%) & ) 26 (49.1\%) & 23 (28.4\%) & 36 (63.2\%) & \\
\hline Black & 49 (57.6\%) & 27 (50.9\%) & 57 (70.4\%) & $21(36.8 \%)$ & \\
\hline Other & $\mathrm{I}(\mathrm{I} .2 \%)$ & $0(0.0 \%)$ & $\mathrm{I}(\mathrm{I} .2 \%)$ & $0(0.0 \%)$ & \\
\hline Marital status & & & & & $0.364^{b}$ \\
\hline Never married & $20(23.5 \%)$ & $3(5.7 \%)$ & 20 (24.7\%) & $4(7.0 \%)$ & \\
\hline Married & 38 (44.7\%) & ) 31 (58.5\%) & 37 (45.7\%) & 30 (52.6\%) & \\
\hline Widowed & $5(5.9 \%)$ & $12(22.6 \%)$ & $3(3.7 \%)$ & $16(28.1 \%)$ & \\
\hline Separated & $6(7.1 \%)$ & $2(3.8 \%)$ & $10(12.3 \%)$ & 2 (3.5\%) & \\
\hline Divorced & $16(18.8 \%)$ & 5 (9.4\%) & II (13.6\%) & 5 (8.8\%) & \\
\hline Employment & & & & & $<0.00 I^{\mathrm{c}}$ \\
\hline Employed & 33 (38.8\%) & ) 8 (I5.1\%) & 35 (43.2\%) & 7 (I2.3\%) & \\
\hline Volunteer & $2(2.4 \%)$ & $2(3.8 \%)$ & $5(6.2 \%)$ & $3(5.3 \%)$ & \\
\hline Retired & 27 (31.8\%) & 43 (8I.1\%) & $20(24.7 \%)$ & $43(75.4 \%)$ & \\
\hline Unemployed & $23(27.1 \%)$ & $0(0.0 \%)$ & 21 (25.9\%) & 4 (7.0\%) & \\
\hline Residence & & & & & 0.459 \\
\hline Rural & 36 (43.4\%) & 16 (30.2\%) & 30 (38.5\%) & 23 (4I.8\%) & \\
\hline Urban & 47 (56.6\%) & ) 37 (69.8\%) & 48 (6I.5\%) & 32 (58.2\%) & \\
\hline \multicolumn{6}{|l|}{$\begin{array}{l}\text { Clinical } \\
\text { (baseline) }\end{array}$} \\
\hline Body mass index & $38.1 \pm 10.2$ & $33.1 \pm 6.8$ & $37.7 \pm 10.0$ & $34.7 \pm 8.0$ & 0.005 \\
\hline Ankle (cm) & $25.1 \pm 5.0$ & $24.4 \pm 3.6$ & $24.4 \pm 3.1$ & $24.9 \pm 4.0$ & 0.634 \\
\hline Calf $(\mathrm{cm})$ & $41.3 \pm 7.2$ & $37.6 \pm 4.6$ & $39.6 \pm 5.4$ & $38.3 \pm 7.0$ & 0.003 \\
\hline CEAP stage 5 & 49 (57.6\%) & 35 (66.0\%) & 45 (55.6\%) & ) 31 (54.4\%) & 0.590 \\
\hline \multicolumn{6}{|l|}{ VEINES score } \\
\hline Total score & $43.2 \pm 20.2$ & $49.8 \pm 21.4$ & $47.8 \pm 21.6$ & $54.7 \pm 24.2$ & 0.024 \\
\hline Symptom score & $44.6 \pm 21.4$ & $53.6 \pm 22.4$ & $49.4 \pm 20.9$ & $57.5 \pm 25.5$ & 0.008 \\
\hline \multicolumn{6}{|c|}{ Medical conditions } \\
\hline Varicose veins & 21 (24.7\%) & 12 (22.6\%) & $22(27.2 \%)$ & ) 19 (33.3\%) & 0.593 \\
\hline Diabetes & $46(54.1 \%)$ & $36(67.9 \%)$ & $48(59.3 \%)$ & $35(6 \mathrm{I} .4 \%)$ & 0.446 \\
\hline Hypertension & $60(70.6 \%)$ & $47(88.7 \%)$ & $53(65.4 \%)$ & $43(75.4 \%)$ & 0.024 \\
\hline Arthritis & $33(38.8 \%)$ & $25(47.2 \%)$ & $24(29.6 \%)$ & $38(66.7 \%)$ & $<0.001$ \\
\hline \multicolumn{6}{|l|}{ Medications } \\
\hline Insulin & $28(32.9 \%)$ & ) $19(35.8 \%)$ & $24(29.6 \%)$ & ) I4 (24.6\%) & 0.591 \\
\hline Diuretics & 15 (I7.6\%) & ) I5 (28.3\%) & 18 (22.2\%) & ) 18 (3I.6\%) & 0.225 \\
\hline Diabetes pill & 33 (38.8\%) & 22 (4I.5\%) & 30 (37.0\%) & $23(40.4 \%)$ & 0.957 \\
\hline Pain pills & 31 (36.5\%) & ) I 4 (26.4\%) & $16(19.8 \%)$ & 19 (33.3\%) & 0.095 \\
\hline Cholesterol & $32(37.6 \%)$ & 30 (56.6\%) & 24 (29.6\%) & 36 (63.2\%) & $<0.001$ \\
\hline Antihypertensive & 55 (64.7\%) & 42 (79.2\%) & 48 (59.3\%) & 34 (59.6\%) & 0.086 \\
\hline
\end{tabular}

Notes: Data are represented as mean \pm standard deviation for continuous variables and number (\%) for categorical variables. ${ }^{a}$ p-value was calculated using only White and Black categories (excluded 2 subjects in "other" category). b $p$-value was calculated using 2 categories used in analysis due to small sample size: married vs not married. c $p$-value was calculated using 2 categories used in analysis due to small sample size: employed vs not employed.

Abbreviation: CEAP, clinical, etiological, anatomical, pathophysiological. 
was included as fixed effect. Potential covariates included demographics (age, sex, race, marital status, employment, and residence) and clinical characteristics (time-varying covariate calf circumference), as well as potential effect modifiers, such as interaction terms of covariates with the group variable. Covariates with a bivariate $p$-value $<0.2$ were included in the full model. Model selection was carried out by sequentially removing the covariate with the highest $p$-value until only the covariates with 0.05 level of significance remained.

\section{Results}

There were 276 participants randomized into this exploratory study, 138 subjects for each arm (cooling and control) (Figure 1). The mean age was 63 years and 61 years for cooling and control $(p=0.207)$, respectively, with approximately half the sample female or married (Table 2). The baseline VEINES scores were lower in the cooling group than the control group (VEINES-QOL score 45.7 vs 50.7, $p=0.064$; VEINES-Sym score 48.0 vs $52.7, p=0.093$ for cooling and control groups, respectively) though the differences did not reach statistical significance.

\section{Aim I}

When the VEINES assessment was analyzed among cooling and control groups (Figure 2A and B), both groups had statistically significant increases in unadjusted mean change VEINES-QOL scores of $15.7(p<0.0001)$ and $15.1(p<0.0001)$ points, respectively, over the 9-month study duration. No

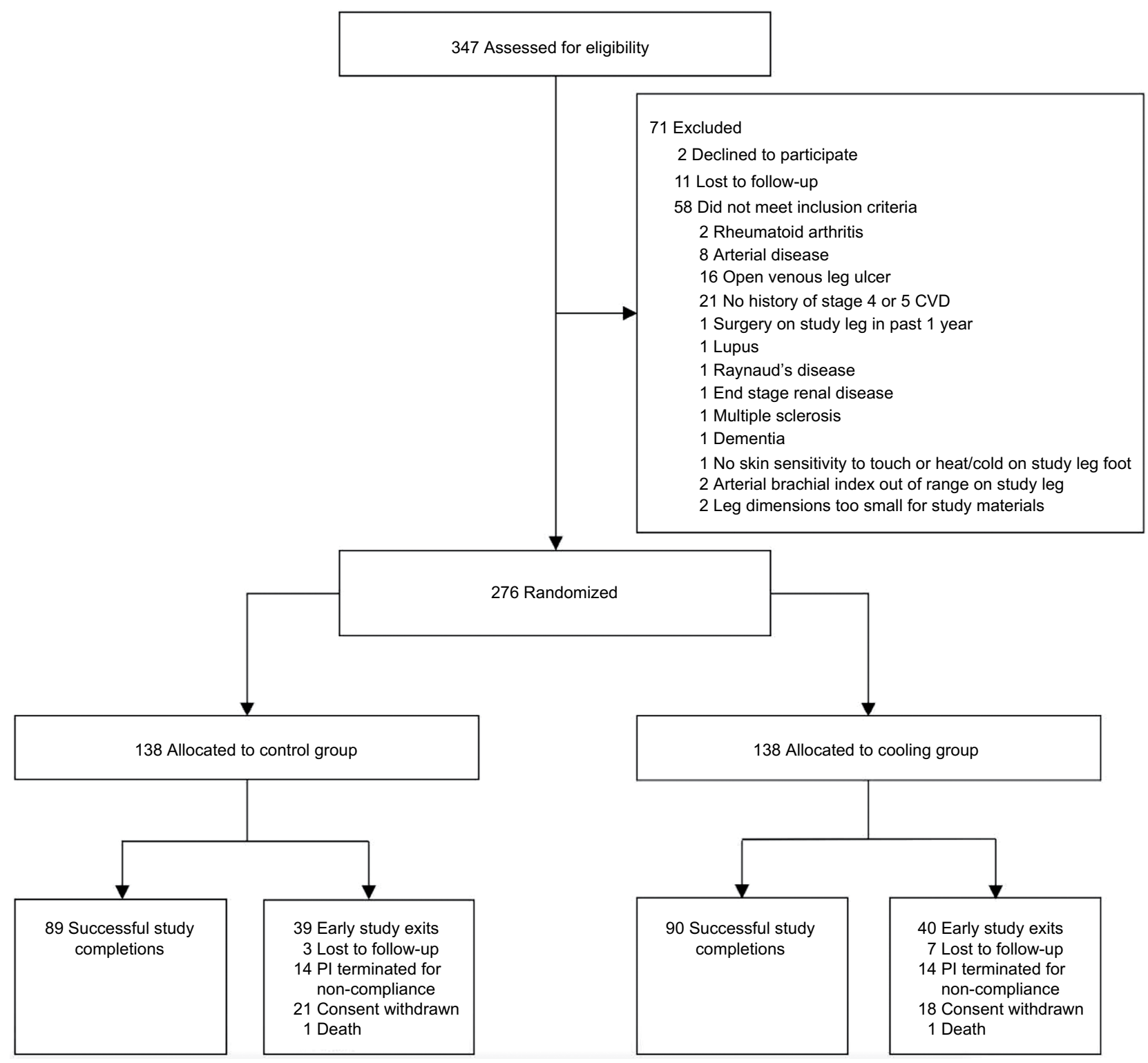

Figure I CONSORT participant diagram depicting the flow of participants through the trail. Abbreviation: PI, Principal investigator. 

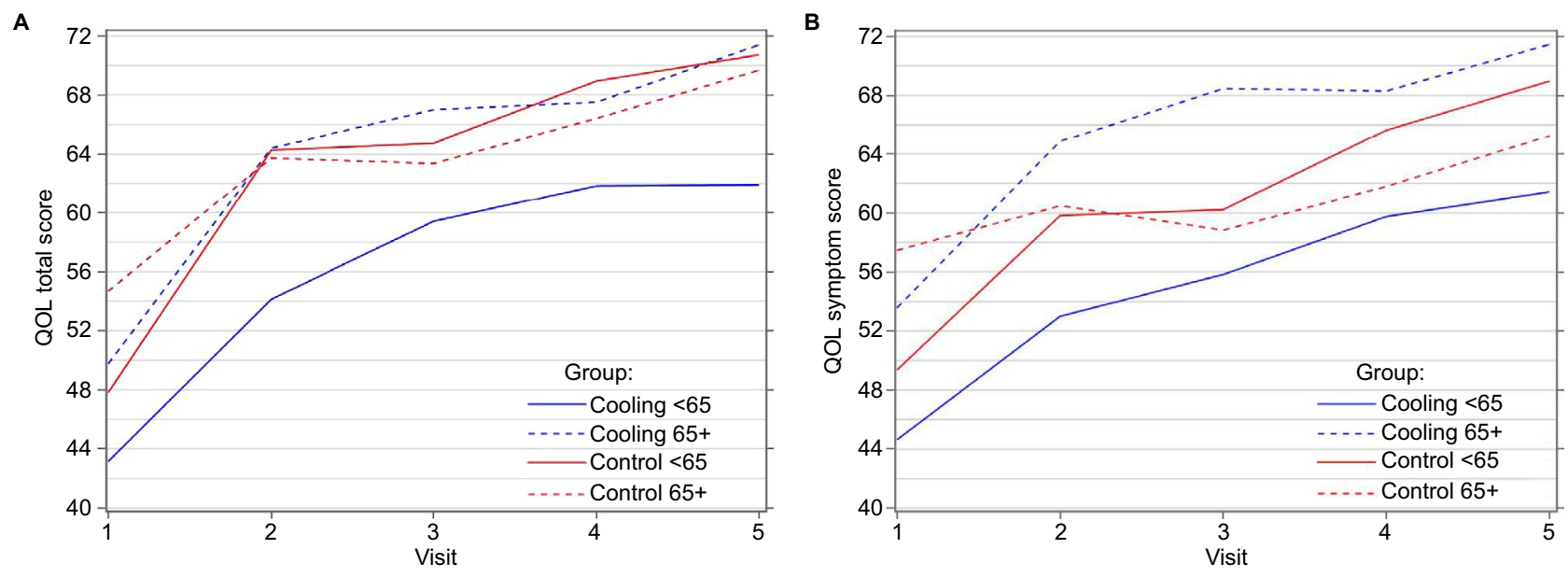

Figure 2 VEINES total scores (A); VEINES symptom scores (B).

Notes: (A) Unadjusted mean VEINES total scores over the 9-month study period by treatment and age group. (B) Unadjusted mean VEINES symptom scores over the 9-month study period by treatment and age group.

Abbreviation: QOL, quality of life.

statistically significant difference in change of QOL was observed between the treatment and control groups $(\Delta=0.69$, $p=0.57)$. Similarly, when adjusting for the influence of covariates in QOL, both cooling and control groups had statistically significant increases in adjusted mean change VEINES-QOL scores $(\Delta=13.5, p<0.0001 ; \Delta=12.8, p<0.0001$, respectively) over the 9-month study duration with no statistically significant difference in change of QOL observed between groups ( $\Delta=0.67, p=0.58)$. However, a statistically significant age-bytreatment interaction $(p<0.0001)$ indicated that the impact of treatment on VEINES-QOL scores was related to age; the change VEINES-QOL score for the cooling group was 0.4 points higher than for someone of the same age in the control group. The change VEINES-QOL score was 5.5 points higher for females than males $(p<0.0001)$ and 6.0 points higher among those married compared to those who were not married $(p<0.0001)$. Those not employed full- or part-time had a change VEINES-QOL score of 9.3 points higher than those who were employed $(p<0.0001)$. Living in rural areas increased the change VEINES-QOL score by 3.8 points as compared to those living in urban areas $(p=0.002)$. For every $3 \mathrm{~cm}$ calf circumference increase from baseline, the VEINESQOL score decreased 1 point from baseline $(p=0.042)$.

Similarly, both cooling and control groups had a statistically significant mean increase in VEINES-Sym scores from baseline $(10.4, p<0.0001 ; 6.7, p<0.0001$ respectively). The cooling group, however, had a significantly higher increase compared to the control group $(\Delta=3.7, p=0.015)$. As with the VEINES-QOL score, the effect of treatment on VEINESSym scores was significantly related to age, indicated by a statistically significant age-by-treatment interaction; the change from baseline VEINES-Sym score for the cooling group was 0.4 points higher compared to someone of the same age in the control group $(p=0.0003)$. Females had higher average change scores in both cooling and control groups (mean $=15.1$ and 7.8, respectively) than men (5.7 and 5.5, respectively), with a statistically significant treatment-by-sex interaction $(p=0.010)$. Among those not employed full- or part-time, the mean change VEINES-Sym score was 12.6 and 12.0 for cooling and control groups, respectively; however, for those employed full- or part-time, the mean change VEINES-Sym score was 8.1 for the cooling group but only 1.4 for the control group, with a statistically significant treatment interaction $(p=0.046)$. Being married was associated with a 6.3-point increase in change from baseline VEINESSym score compared to those not married $(p<0.0001)$, and $1 \mathrm{~cm}$ increase from baseline in calf circumference decreased the VEINES-Sym score by 0.4 points $(p=0.021)$.

Given the strong age by treatment association in VEINES scores, for subsequent analyses, subjects in each treatment group were categorized by age as those less than 65 years and those greater than or equal to 65 years. For the cooling group, there were $85(61.6 \%)$ participants $<65$ years of age and $53(38.4 \%)$ participants $\geq 65$, with mean age of 55 and 74 years, respectively; in the control group, there were 81 (58.7\%) participants $<65$ years of age and $57(41.3 \%$ ) participants $\geq 65$ years or older, with mean age of 53 and 72 years, respectively.

\section{Aim 2}

There was a statistically significant difference in the distribution of race between groups ( $p=0.001$, Table 3 ). In the cooling group, the distribution was similar to the overall sample, with approximately half of the participants being African American/ Black in both the age groups, $<65$ and $\geq 65$ years. However, in 
Table 3 Change from baseline to month 9 in VEINES-QOL scores by treatment and age group (least squared means)

\begin{tabular}{|c|c|c|c|}
\hline Scores & Cooling & Control & $p$ (between treatments) \\
\hline \multicolumn{4}{|l|}{ Total score ${ }^{a}$} \\
\hline Total sample & $13.5^{*}$ & $12.8^{*}$ & 0.576 \\
\hline$<65$ years & $13.9 *$ & $18.3^{*}$ & 0.007 \\
\hline \multirow[t]{2}{*}{$\geq 65$ years } & $10.8^{*}$ & $4.5^{* *}$ & $<0.001$ \\
\hline & $<65$ years & $\geq 65$ years & $p$ (between age groups) \\
\hline Cooling & $13.8^{*}$ & $14.7^{*}$ & 0.602 \\
\hline Control & $17.7^{*}$ & $5.8 * * *$ & $<0.0001$ \\
\hline \multicolumn{4}{|c|}{ Symptom score ${ }^{a}$} \\
\hline Total sample & $10.4^{*}$ & $6.7^{*}$ & 0.015 \\
\hline$<65$ years & $11.3^{*}$ & $12.4^{*}$ & 0.540 \\
\hline \multirow[t]{2}{*}{$\geq 65$ years } & $8.1^{*}$ & -1.0 & $<0.0001$ \\
\hline & $<65$ years & $\geq 65$ years & p (between age groups) \\
\hline Cooling & $10.7^{*}$ & $13.7^{*}$ & 0.112 \\
\hline Control & $12.5^{*}$ & 0.7 & $<0.0001$ \\
\hline
\end{tabular}

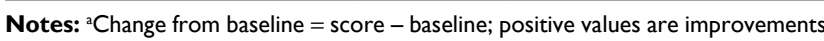
and negative values are declines. Change from baseline $p$-value: $*<0.000 \mathrm{I}, * *<0.01$, $* * *<0.001$.

Abbreviation: QOL, quality of life.

the control group, more than two-thirds of the age $<65$ years group were African American/Black (70.4\%), whereas almost two-thirds of the age $\geq 65$ years group were White $(63.2 \%)$.

As expected, employment status was statistically significantly different between age groups: a small proportion of the $\geq 65$ age groups were employed in both treatment groups compared to the $<65$ age groups (employed $15.1 \%$ and $12.3 \%$ for the $\geq 65$ age group in cooling and control groups, respectively; $p<0.0001)$. There were statistically significant differences in BMI and calf circumference $(\mathrm{cm})$ between groups, with the $<65$ age group having higher BMI $(p=0.0045)$ and higher average calf circumference $(p=0.0028)$ compared to the $\geq 65$ age group.

\section{Aim 3}

\section{Age $<65$ group}

When the VEINES-QOL change from baseline score was compared between treatment groups for those $<65$ years old, there were statistically significant increases in average change from baseline for VEINES-QOL and VEINES-Sym scores for both groups (all $p<0.0001$, Table 4 ), with the highest increases in the control group.

The average change from baseline in VEINES-QOL score for cooling and control was 13.9 and 18.3, respectively, with a significantly larger increase in the control group $(\Delta=-4.4$, $p=0.007$ ). Those participants who were not employed at baseline had a change from baseline score 7.3 points higher than those participants who were employed $(p<0.0001)$. The change VEINES-QOL score was higher for females than males $(6.0, p=0.0003)$ and those living in rural areas compared to urban areas $(5.3, p=0.0012)$. For every $1 \mathrm{~cm}$
Table 4 Demographic features of sample at baseline

\begin{tabular}{|c|c|c|c|}
\hline Demographics & $\begin{array}{l}\text { Cooling } \\
n=138\end{array}$ & $\begin{array}{l}\text { Control } \\
n=138\end{array}$ & $p$-value \\
\hline Age (years) & $62.7 \pm 11.5$ & $60.9 \pm 11.9$ & 0.207 \\
\hline Sex & & & 0.468 \\
\hline Male & $66(48.2 \%)$ & $60(43.5 \%)$ & \\
\hline Female & $72(52.2 \%)$ & $78(56.5 \%)$ & \\
\hline Race & & & $0.808^{\mathrm{a}}$ \\
\hline White & $6 \mathrm{I}(44.5 \%)$ & 59 (42.8\%) & \\
\hline Black & $76(55.1 \%)$ & $78(56.5 \%)$ & \\
\hline Other & $\mathrm{I}(0.7 \%)$ & $\mathrm{I}(0.7 \%)$ & \\
\hline Marital status & & & 0.802 \\
\hline Never married & $23(16.7 \%)$ & $24(17.4 \%)$ & \\
\hline Married & 69 (50.0\%) & 67 (48.6\%) & \\
\hline Widowed & 17 (I2.3\%) & 19 (I3.8\%) & \\
\hline Separated & $8(5.8 \%)$ & $12(8.7 \%)$ & \\
\hline Divorced & $2 I(I 5.2 \%)$ & $16(11.6 \%)$ & \\
\hline Employment & & & 0.616 \\
\hline Employed & $4 \mathrm{I}(29.7 \%)$ & $42(30.4 \%)$ & \\
\hline Other & $4(2.9 \%)$ & $8(5.8 \%)$ & \\
\hline Retired & 70 (50.7\%) & $63(45.7 \%)$ & \\
\hline Unemployed & $23(16.7 \%)$ & $25(18.1 \%)$ & \\
\hline Residence & & & 0.786 \\
\hline Rural & $52(38.2 \%)$ & $53(39.8 \%)$ & \\
\hline Urban & $84(61.8 \%)$ & $80(60.2 \%)$ & \\
\hline \multicolumn{4}{|l|}{ Clinical (baseline) } \\
\hline Body mass index & $36.2 \pm 9.3$ & $36.4 \pm 9.3$ & 0.819 \\
\hline Ankle $(\mathrm{cm})$ & $24.9 \pm 4.5$ & $24.6 \pm 3.5$ & 0.560 \\
\hline Calf $(\mathrm{cm})$ & $39.9 \pm 6.6$ & $39.1 \pm 6.1$ & 0.306 \\
\hline CEAP stage 5 & $84(60.9 \%)$ & $76(55.1 \%)$ & 0.329 \\
\hline \multicolumn{4}{|l|}{ VEINES-QOL score } \\
\hline Total score & $45.7 \pm 20.9$ & $50.7 \pm 22.7$ & 0.064 \\
\hline Symptom score & $48.0 \pm 22.2$ & $52.7 \pm 23.2$ & 0.093 \\
\hline \multicolumn{4}{|l|}{ Medical conditions } \\
\hline Varicose veins & $33(23.9 \%)$ & $4 \mathrm{I}(29.7 \%)$ & 0.277 \\
\hline Diabetes & $82(59.4 \%)$ & $83(60.1 \%)$ & 0.902 \\
\hline Hypertension & 107 (77.5\%) & $96(69.6 \%)$ & 0.133 \\
\hline Osteoarthritis & $58(42.0 \%)$ & 62 (44.9\%) & 0.627 \\
\hline \multicolumn{4}{|l|}{ Medications } \\
\hline Insulin & 47 (34.1\%) & $38(27.5 \%)$ & 0.241 \\
\hline Diuretics & $30(21.7 \%)$ & $36(26.1 \%)$ & 0.397 \\
\hline $\begin{array}{l}\text { Oral antidiabetic } \\
\text { agents }\end{array}$ & 55 (39.9\%) & 53 (38.4\%) & 0.805 \\
\hline Pain medications & $45(32.6 \%)$ & $35(25.4 \%)$ & 0.185 \\
\hline Statins & 62 (44.9\%) & $60(43.5 \%)$ & 0.808 \\
\hline Antihypertensives & 97 (70.3\%) & $82(59.4 \%)$ & 0.059 \\
\hline
\end{tabular}

Notes: Data are represented as mean \pm standard deviation for continuous variables and number (\%) for categorical. a $p$-value ( $\mathrm{P}$ ) was calculated using only White and Black ethnic categories (excluded 2 subjects in "other" category).

Abbreviation: CEAP, clinical, etiological, anatomical, pathophysiological.

ankle circumference increase from baseline, there was a 0.6point increase in change from baseline total score $(p=0.025)$.

The average change from baseline in VEINES-Sym score for the cooling and control groups was 11.3 and 12.4, respectively $(\Delta=-1.1, p=0.540)$. Among those not employed full- or part-time, the mean change VEINES-Sym score was 12.0 and 17.1 points for cooling and control groups, respectively; for 
those employed full- or part-time, the mean change VEINESSym score was 10.6 and 7.6 for cooling and control groups, respectively (treatment-by-employment, $p=0.025$ ). The change VEINES-Sym score was higher for females than males $(\Delta=6.2, p=0.001)$, those who were married compared to not married $(\Delta=5.0, p=0.007)$, and those living in rural areas compared to urban areas $(\Delta=5.5, p=0.003)$.

\section{Age $\geq 65$ group}

When the VEINES-QOL change from baseline score was analyzed for those $\geq 65$ years old, there were statistically significant increases in average change from baseline of VEINES-QOL scores for both cooling and control groups ( $p<0.0001$ and $p=0.004$, respectively); however, average change in VEINES-Sym score for cooling increased $(p<0.0001)$, while for the control group it decreased $(p=0.557)$. Contrary to the age $<65$ group, the highest increases were seen in the cooling group.

The average change from baseline in VEINES-QOL score for cooling and control groups was 10.8 and 4.5, respectively ( $\Delta=6.3, p=0.0004)$. Those participants who were not employed ( $85 \%$ in this age group) at baseline had a change score 14.3 points higher than those participants who were employed $(p<0.0001)$. The change VEINES-QOL score was 10.3 points higher for those who were married than those not married $(p<0.0001)$. For every $1 \mathrm{~cm}$ calf circumference increase from baseline, there was a 0.6-point decrease in change from baseline VEINES-QOL score $(p=0.010)$.

The average change from baseline in VEINES-Sym score for the cooling and control groups was 8.1 and -1.0 , respectively $(\Delta=9.1, p<0.0001)$. Among females, the mean change VEINES-Sym score was 14.0 and -0.3 points for cooling and control groups, respectively; for men, the mean change VEINES-Sym score was 2.2 and -1.8 for cooling and control groups, respectively $(p=0.001)$. The change VEINES-Sym score was higher for those not employed full or part-time compared to those employed full or part-time (13.2, $p<0.0001)$ and those who were married compared to not married $(9.9, p<0.0001)$. A $1 \mathrm{~cm}$ increase from baseline in calf circumference decreased the VEINES-Sym score by 0.7 points from baseline $(p=0.009)$.

\section{Cooling group}

When the change from baseline scores was compared between age groups for those in the cooling arm, statistically significant increases in average change from baseline were observed for VEINES-QOL scores and VEINES-Sym scores for both age groups (all $p<0.0001$, Table 3 ), with the highest increases in the $\geq 65$ age group for both scores. However, there were no statistically significant differences in the mean change from baseline scores between age groups ( $p=0.60$ and $p=0.11$ for $<65$ and $\geq 65$ age groups, respectively).

\section{Control group}

Similarly, comparison of change from baseline scores between age groups for those in the control group showed statistically significant increases in average change from baseline for VEINES-QOL scores for both groups, with a statistically significantly higher increase among those in the $<65$ age group compared to $\geq 65$ age group $(\Delta=11.9, p<0.0001)$. VEINES-Sym score for both age groups increased; however, only the $<65$ years group had a statistically significant change from baseline score $(p<0.0001)$, which was statistically significantly higher compared to change from baseline for the $\geq 65$ years group $(\Delta=11.8, p<0.0001)$.

\section{Discussion}

The results of this exploratory study of 276 individuals with CEAP stages C4 (skin damage) and C5 (history of VLUs) suggest that the cooling intervention, coupled with standard of care, improved QOL. The greatest improvements were observed in older individuals, females, those who were married, not working full time, and living in rural areas. There were no associations noted with comorbid conditions such as diabetes, suggesting individuals with comorbid conditions experienced no differences in QOL between groups.

In aim 1, differences between the cooling and control groups on QOL were examined. It is well recognized that lower QOL is prevalent in individuals with CVD. ${ }^{1-3,16,24}$ The symptoms of burning, swelling, pain, heaviness, and achiness lead to ongoing disability and impaired function in daily activities. ${ }^{16}$ However, recent research of nonpharmacological options is limited for the self-management of CVD symptoms that would augment established standard of care that includes compression and leg elevation. Compression has been shown to positively influence QOL, but unfortunately adherence is low. ${ }^{25}$ In our study, adherence was moderately high, and both treatment and control groups demonstrated improvements with standard of care over time, with individuals using the cooling cuff reporting higher scores for both QOL and symptoms. Older individuals in the cooling group, $\geq 65$ years of age in particular, experienced much greater improvements in symptoms compared to the older control group $\geq 65$ years of age; the latter group experienced no improvements at all, in fact, they reported a worsening of symptoms from baseline. Older individuals may be more 
susceptible to the physiological and psychological benefits of cooling. Between treatment groups and age, females were more positively susceptible to the cooling intervention for improvements in QOL and symptom outcomes, as were individuals who were not employed or working part-time, married, and living in rural areas.

Baseline characteristics of the participants reflect the clinical-anatomical-etiological pathogenesis of CVD; the disease affects older individuals and females. Our population had worse disease stages and several comorbid conditions, was older, with a mean age of 62 years, and had a slightly higher proportion $(54.4 \%)$ of females, all factors highly associated with poorer QOL. Older age and being female are risk factors for CVD, and epidemiological studies suggest females are more likely to experience all stages of CVDs, from varicose veins to leg ulcers, and have lower QOL and greater severity of symptoms. ${ }^{1,2,11,16,26-28}$ It is important to note that in our study, we wished to assess change over time in response to the cooling treatment; while we assessed QOL at baseline, it was not our intent to determine whether QOL was poor or low at that time. The VEINES-QOL instrument was selected to provide a reliable and responsive measure of changes over time; it did not provide a score that characterized the level of QOL. We sought to determine how much change in QOL and symptoms took place over the study period, not whether the changes were related to alterations in the disease process. ${ }^{22}$

It is generally known that differences exist between males and females with regard to thermal sensitivity. ${ }^{29}$ Regardless of age, females display a greater thermal sensitivity than males; however, age-related declines in sensitivity between both older males and females exist. ${ }^{30}$ Furthermore, younger and older females experience lower skin temperatures in response to the same cooling stimuli suggesting that females are more sensitive to cooling. Females have more peripheral skin receptors, and those with CVD are noted to have different symptom presentations than males. Older females are reported to have more neuropathic-type complaints such as itching, burning, throbbing, and night cramps compared to males who report more nocioceptive symptoms such as heavy and achy legs. ${ }^{31,32}$

The influence of sex hormones and phases of the luteal cycle may have explained some of the variation between sexes in younger females although recent data suggest that there are no intramenstrual differences noted in response to cooling the feet. ${ }^{33}$ However in our study, it was assumed that the majority of females were postmenopausal (average age of females was 61.4 years, range 34-87), thus hormonal influences were not likely to explain why females, particularly older females, reported improvements in QOL and a more pronounced reduction in negative symptoms, related to cooling. Behaviorally, this sex response in older females could be partially explained by females initiating a cognitive temperature regulation response earlier than males in order to maintain thermal balance. $.^{34} \mathrm{How}-$ ever, the relationship between this response and cold perception for relief of skin symptoms is not well documented. Through various cutaneous sensory and behavioral mechanisms, females respond differently to cooling, and these data suggest low level cooling of skin affected by CVD provides positive outcomes related to symptom reduction and improvements in QOL. Physiologically via these pathways, cooling legs in females provided greater "relief" from these neuropathic-type symptoms and improved QOL.

Research has shown that subjective health is related to numerous external factors such as social support, marital status, and location of residence, in particular, neighborhood characteristics. ${ }^{35,36}$ Marriage provides protective health benefits, and married individuals have longer survival times and lower incidence of health conditions compared to unmarried individuals. ${ }^{37}$ Marital status has also been shown to positively affect QOL, but this differs by sex and age.$^{38}$ Having a spouse/partner as a social support may provide encouragement and reminders to engage in self-management behaviors that improve overall well-being. This might have been the case with the participants in the study. No clear relationship between residing in urban or rural environments and QOL has been established for individuals with CVD. Data suggest that individuals living in rural areas may have less access to health care, but research on the influence on QOL is lacking. ${ }^{39}$ In the current study, individuals in both age and treatment groups residing in rural areas reported improvements in QOL and symptoms with cooling. It has been suggested that individuals living in rural areas may have a greater sense of satisfaction or gratification with life and have well-established relationships within the community, and also visiting with friends, neighbors, and relatives has a stronger positive effect on subjective well-being. ${ }^{40-43}$ Satisfaction with living conditions in rural settings may have bolstered the addition of cooling to standard of care in providing improved QOL and symptom reduction such that participants felt a greater sense of control and perceived well-being in overall health, ${ }^{44}$ although control and well-being were not study outcomes.

In aim 2, it was no surprise that individuals who were employed were $<65$ years of age and reported less improvement in QOL and reduction in symptoms compared to their nonworking older counterparts. Many of those who worked 
reported being in jobs that required long hours of standing and had higher BMIs and were obese $\left(38.1 \mathrm{~kg} / \mathrm{m}^{2}\right.$ for the treatment group and $37.7 \mathrm{~kg} / \mathrm{m}^{2}$ for the control group). Standing for long periods of time increases complaints related to CVD. ${ }^{45} \mathrm{We}$ found that cooling of legs of participants with larger calf circumference, also indicative of obesity and/or swelling, resulted in minimal improvements in QOL or symptom reduction. In general, individuals with swelling have reduced function and higher reports of pain and disability. ${ }^{46-48}$ Our findings are consistent with those reported in the literature and suggest working individuals may be less compliant with therapy. A limitation of the study was the unequal distribution of race in the control groups. Our randomization scheme did not consider stratified allocation to prevent uneven race representation, and consequently there were higher number of Black/African American participants in the younger control group and White participants in the older age control group. Thus, comparisons among race by treatment by age were unable to be conducted.

In aim 3, individuals in the older age group had a very positive QOL response to cooling. There is age-dependent neural degeneration within the afferent signals from reduced peripheral thermoreceptor density, reduced conduction velocity, and/or decreased neurotransmitter release. ${ }^{49}$ These changes are posited to be responsible for the decline in sensitivity and an increase in neuropathic-type sensations. ${ }^{49}$ It is currently unknown whether age-related changes and the interaction with sex on sudomotor and cutaneous vascular function, both important to maintain thermal balance, are involved in thermal sensitivity and perception. ${ }^{6,30}$ The cooling cuff used in this study reduced skin temperature by $2-5^{\circ} \mathrm{C}$ for approximately 30 minutes, providing a cooling sensation that affects afferent sensory perception in various skin neuronal pathways. The negative symptoms of itching, burning, or tingling were reduced. These findings are consistent with studies in which cooling relieved itching sensations in various skin disorders with an inflammatory component such as CVD. ${ }^{50}$

\section{Limitations}

One of the major limitations of this trial was recruitment of the population; they experienced multiple chronic conditions and had other vascular disorders including arterial insufficiency, which excluded them from the trial. Data obtained from the exit survey suggested some of the participants found the cuff to be "too cold", "covered too much of my leg", and "was heavy"; thus, it was unclear whether they kept the cuff on for the required amount of time needed to effectively achieve the desired cooling results. Conversely, other participants reported the cuff "felt so good"; some indicated they used it more frequently than the protocol required. Whether this increased frequency of use affected outcomes is not known. Measuring temperatures three times daily during active treatment was also considered burdensome by some participants, but most stated they liked knowing their leg temperatures over time and knew this was an important aspect of the study. These comments informed the revision of procedures for an ongoing randomized controlled trial of a small cooling patch, compared to placebo, in a "real-world" trial in which participants receive the patch and are instructed to take care of their legs and feet per instruction from the primary care provider. QOL and self efficacy are two outcomes of the study.

\section{Conclusion}

CVD remains a poorly controlled chronic condition and has a major negative influence on QOL. It is important for health care providers to assess for the presence of deleterious symptoms including burning, heaviness, and cramps as these factors can lead to low function and psychosocial impairments such as poor sleep, anxiety, and depression. Data from this descriptive study suggest that cooling the skin when used in conjunction with compression leggings and leg elevation provides a preliminary signal of efficacy on improved QOL. However, findings are preliminary and need to be confirmed in future studies. For clinical care, we recommend every other day cooling with a non-solid-gel pack encased in some type of thin cloth and placed on the affected skin with legs elevated on at least two pillows for 30 minutes. Compression leggings are to be worn when individuals are ambulatory and removed during the cooling treatment. There continues to be a tremendous need for training of health care professionals on best practices for managing CVDs and the debilitating symptoms in order to improve the QOL of these individuals.

\section{Acknowledgments}

The authors thank Dr Jane Zapka ScD for the assistance on the research design and editorial guidance. This study was funded by the National Institute of Nursing Research (NINR) Award \# R01NR012237 and support from the South Carolina Clinical \& Translational Research (SCTR) Institute, with an academic home at the Medical University of South Carolina, through National Institutes of Health (NIH) grant number UL1 TR000062. The use of REDCap was supported by NIH/National Center for Advancing Translational Sciences (NCATS) UL1TR000062. The ideas and opinions expressed herein are those of the authors and not necessarily reflective of the NINR.

\section{Disclosure}

The authors report no conflicts of interest in this work. 


\section{References}

1. Lozano Sanchez FS, Sanchez Nevarez I, Gonzalez-Porras JR, et al. Quality of life in patients with chronic venous disease: influence of the socio-demographical and clinical factors. Int Angiol. 2013;32(4): 433-441.

2. Tracz E, Zamojska E, Modrzejewski A, Zaborski D, Grzesiak, W. Quality of life in patients with venous stasis ulcers and others with advanced venous insufficiency. Holist Nurs Pract. 2015;29(2):96-102.

3. Wachholz PA, Masuda PY, Nascimento DC, Taira CM, Cleto NG. Quality of life profile and correlated factors in chronic leg ulcer patients in the mid-west of Sao Paulo State, Brazil. An Bras Dermatol. 2014;89(1):73-81.

4. Matic P, Jolic S, Tanaskovic S, et al. Chronic venous disease and comorbidities. Angiology. 2015;66(6):539-554.

5. Kelechi TJ, Mueller M, Dooley M. Sex differences in symptom severity and clusters in patients with stage $\mathrm{C} 4$ and stage $\mathrm{C} 5$ chronic venous disease. Eur J Cardiovasc Nurs. 2017;16(1):28-36.

6. Do HT, Edwards H, Finlayson, K. Identifying relationships between symptom clusters and quality of life in adults with chronic mixed venous and arterial leg ulcers. Int Wound J. 2016;13(5):904-911.

7. Gonzalez de la Torre H, Quintana-Lorenzo ML, Perdomo-Perez E, Verdu J. Correlation between health-related quality of life and venous leg ulcer's severity and characteristics: a cross-sectional study. Int Wound J. 2017;14(2):360-368.

8. Green J, Jester R, McKinley R, Pooler A. The impact of chronic venous leg ulcers: a systematic review. J Wound Care. 2014;23(12):601-612.

9. Gonzalez-Consuegra RV, Verdu J. Quality of life in people with venous leg ulcers: an integrative review. J Adv Nurs. 2011;67(5):926-944.

10. Hopman WM, Buchanan M, VanDenKerkhof EG, Harrison MB. Pain and health-related quality of life in people with chronic leg ulcers. Chronic Dis Inj Can. 2013;33(3):167-174.

11. Bryan Romic R, Bryan A, Romic I, Cvitanovic H, Duvancic T, LugovicMihic L. Quality of life and perception of disease in patients with chronic leg ulcer. Acta clinica Croatica. 2015;54(3):309-314.

12. Rabe E, Agus GB, Roztocil K. Analysis of the effects of micronized purified flavonoid fraction versus placebo on symptoms and quality of life in patients suffering from chronic venous disease: from a prospective randomized trial. Int Angiol. 2015;34(5):428-436.

13. Tollow P, Ogden J, Whiteley MS. The comparative impact of conservative treatment versus superficial venous surgery, for the treatment of venous leg ulcers: a systematic review of the impact on patients' quality of life. Phlebology. 2016;31(2):82-93.

14. Lloret P, Redondo P, Cabrera J, Sierra A. Treatment of venous leg ulcers with ultrasound-guided foam sclerotherapy: healing, longterm recurrence and quality of life evaluation. Wound Repair Regen. 2015;23(3):369-378.

15. O'Brien J, Finlayson K, Kerr G, Edwards H. Evaluating the effectiveness of a self-management exercise intervention on wound healing, functional ability and health-related quality of life outcomes in adults with venous leg ulcers: a randomised controlled trial. Int Wound J. 2017;14(1):130-137.

16. Ceviker K, Sahinalp S, Cicek E, et al. Quality of life in patients with chronic venous disease in Turkey: influence of different treatment modalities at 6-month follow-up. Qual Life Res. 2016;25(6):1527-1536.

17. Bharara M, Viswanathan V, Cobb JE. Cold immersion recovery responses in the diabetic foot with neuropathy. Int Wound J. 2008;5(4):562-569.

18. Kelechi TJ, Mueller M, Madisetti M, Prentice MA, Dooley MJ. Does cryotherapy improve skin circulation compared to compression and elevation in preventing venous leg ulcers. Int Wound J. Epub 2014 Aug 10.

19. Shibuya N, Schinke TL, Canales MB, Yu GV. Effect of cryotherapy devices in the postoperative setting. J Am Podiatr Med Assoc. 2007; 97(6):439-446.

20. Gloviczki P, Comerota AJ, Dalsing MC, et al. The care of patients with varicose veins and associated chronic venous diseases: clinical practice guidelines of the Society for Vascular Surgery and the American Venous Forum. J Vasc Surg. 2011;53(Suppl 5):S2-S48.
21. Lamping DL, Schroter S, Kurz X, Kahn SR, Abenhaim L. Evaluation of outcomes in chronic venous disorders of the leg: development of a scientifically rigorous, patient-reported measure of symptoms and quality of life. JVas Surg. 2013;37(2):410-419.

22. Kelechi TJ, Mueller M, Zapka JG, King DE. The effect of a cryotherapy gel wap on the microcirculation of skin affected by chronic venous disorders. J Adv Nurs. 2011;67(11):2337-2349.

23. Bland JM, Dumville JC, Ashby RL, et al. Validation of the VEINESQOL quality of life instrument in venous leg ulcers: repeatability and validity study embedded in a randomised clinical trial. BMC Cardiovasc Disord. 2015;15:85.

24. Moura RM, Goncalves GS, Navarro TP, Britto RR, Dias RC. Relationship between quality of life and the CEAP clinical classification in chronic venous disease. Rev Bras Fisioter. 2010;14(2):99-105.

25. Andreozzi GM, Cordova R, Scomparin MA, et al. Effects of elastic stocking on quality of life of patients with chronic venous insufficiency. An Italian pilot study on Triveneto Region. Int Angiol. 2005;24(4);325-329.

26. Escudero Rodriguez JR, Fernandez Quesada F, Bellmunt Montoya S. Prevalence and clinical characteristics of chronic venous disease in patients seen in primary care in Spain: results of the international study Vein Consult Program. Cir Esp. 2014;92(8):539-546.

27. Lohr JM, Bush RL. Venous disease in women: epidemiology, manifestations, and treatment. J Vasc Surg. 2013;57(Suppl 4):S37-S45.

28. Wellborn J, Moceri JT. The lived experiences of persons with chronic venous insufficiency and lower extremity ulcers. $J$ Wound Ostomy Continence Nurs. 2014;41(2):122-126.

29. Gerrett N, Ouzzahra Y, Coleby S, et al. Thermal sensitivity to warmth during rest and exercise: a sex comparison. Eur J Appl Physiol. 2014;114(7):1451-1462.

30. Inoue $\mathrm{Y}$, Gerrett $\mathrm{N}$, Ichinose-Kuwahara $\mathrm{T}$, et al. Sex differences in age-related changes on peripheral warm and cold innocuous thermal sensitivity. Physiol Behav. 2016;164(Pt A):86-92.

31. Yim E, Vivas A, Maderal A, Kirsner RS. Neuropathy and ankle mobility abnormalities in patients with chronic venous disease. JAMA Dermatol. 2014;150(4):385-389.

32. Paul JC, Pieper B, Templin TN. Itch: association with chronic venous disease, pain, and quality of life. J Wound Ostomy Continence Nurs. 2011;38(1):46-54.

33. Lunt H, Tipton M. Differences in conductive foot cooling: a comparison between males and females. Eur J Appl Physiol. 2014;114(12): 2635-2644.

34. Ksenija C, Mark M, Zare F. Cutaneous microvascular response during local cold exposure - the effect of female sex hormones and cold perception. Microvasc Res. 2016;108:34-40.

35. Zheng H, Thomas PA. Marital status, self-rated health, and mortality: overestimation of health or diminishing protection of marriage? J Health Soc Behav. 2013;54(1);128-143.

36. Benyamini Y. Why does self-rated health predict mortality? An update on current knowledge and a research agenda for psychologists. Psychol Health. 2011;26(11):1407-1413.

37. August KJ, Sorkin DH. Marital status and gender differences in managing a chronic illness: the function of health-related social control. Soc Sci Med. 2010;71(10):1831-1838.

38. Han KT, Park EC, Kim JH, Kim SJ, Park S. Is marital status associated with quality of life? Health Qual Life Outcomes. 2014;12:109.

39. Goodridge D, Lawson J, Rennie D, Marciniuk D. Rural/urban differences in health care utilization and place of death for persons with respiratory illness in the last year of life. Rural Remote Health. 2010;10(2):1349.

40. Carta MG, Aguglia E, Caraci F, et al. Quality of life and urban/rural living: preliminary results of a community survey in Italy. Clin Pract Epidemiol Ment Health. 2012;8:169-174.

41. Mair CA, Thivierge-Rikard RV. The strength of strong ties for older rural adults: regional distinctions in the relationship between social interaction and subjective well-being. Int J Aging Hum Dev. 2010;70(2):119-143.

42. Li C, Chi I, Zhang X, Cheng Z, Zhang L, Chen G. Urban and rural factors associated with life satisfaction among older Chinese adults. Aging Ment Health. 2015;19(10):947-954. 
43. Urosevic J, Odovic G, Rapaic D, Davidovic M, Trgovcevic S, Milovanovic V. Quality-of life of the elderly in urban and rural areas in Serbia. Vojnosanit Pregl. 2015;72(11):968-974.

44. Abas MA, Punpuing S, Jirapramupitak T, Tangchonlatip K, Leese M. Psychological wellbeing, physical impairments and rural aging in a developing country setting. Health Qual Life Outcomes. 2009;7:66.

45. Campbell WB, Decaluwe H, Boecxstaens V, et al. The symptoms of varicose veins: difficult to determine and difficult to study. Eur J Vasc Endovasc Surg. 2007;34(6):741-744.

46. Blazek C, Amsler F, Blaettler W, Keo HH, Baumgartner I, Willenberg T. Compression hosiery for occupational leg symptoms and leg volume: a randomized crossover trial in a cohort of hairdressers. Phlebology. 2013;28(5):239-247.
47. dos Santos Crisostomo RS, Costa DS, de Luz Belo Martins C, Fernandes TI, Armada-da-Silva PA. Influence of manual lymphatic drainage on healthrelated quality of life and symptoms of chronic venous insufficiency: a randomized controlled trial. Arch Phys Med Rehabil. 2015;96(2):283-291.

48. Guex JJ, Enrici E, Boussetta S, Avril L, Lis C, Taieb C. Correlations between ankle circumference, symptoms, and quality of life demonstrate the clinical relevance of minimal leg swelling reduction: results of a study in 1,036 Argentinean patients. Dermatol Surg. 2008;34(12):1666-1675.

49. Verdu E, Ceballos D, Vilches JJ, Navarro X. Influence of aging on peripheral nerve function and regeneration. J Peripher Nerv Syst. 2000;5(4):191-208.

50. Park B, Kim SJ. Cooling the skin: understanding a specific cutaneous thermosensation. J Lifestyle Med. 2013;3(2):91-97.
Chronic Wound Care Management and Research

\section{Publish your work in this journal}

Chronic Wound Care Management and Research is an international, peer reviewed, open access, online journal publishing original research, reviews, editorials, and commentaries on the causes and management of chronic wounds and the major issues related to chronic wound management. Topics also include chronic wounds as comorbidities to other

\section{Dovepress}

conditions, patient adherence to therapy, and the economic burden of chronic wounds. The manuscript management system is completely online and includes a very quick and fair peer review system, which is all easy to use. Visit http://www.dovepress.com/testimonials.php to read real quotes from published authors.

Submit your manuscript here: https://www.dovepress.com/chronic-wound-care-management-and-research-journal 